\title{
Physics and Structure of the Galactic disc(s)
}

\author{
Ralph A. Schönrich \\ Max Planck Institute for Astrophysics, \\ Garching, D-85741, Garching, Germany \\ email: rasch@mpa-garching.mpg.de
}

\begin{abstract}
The model of Schönrich \& Binney (2009) offers new ways to understand the chemokinematic structure of the solar neighbourhood in the light of radial mixing. The combination of chemical information with rich kinematic data reveals a still hardly explored abundance of interconnections and structures from which we can learn about both the physics and history of our Galaxy. Large upcoming datasets can be used to improve estimates of central parameters, to shed light on the Galaxy's history and to explore the unexpected way of understanding the well-known division of the Galactic disc yielded by the new model.
\end{abstract}

Keywords. Galaxy: structure, Galaxy: evolution, Galaxy: abundances, Galaxy: kinematics and dynamics

Radial mixing has been a vastly neglected process in modelling galactic discs until it was shown to be crucial for disc evolution from a theoretical point of view by Sellwood \& Binney (2002). Schönrich \& Binney (2009) demonstrated that under regular assumptions about star formation and disc structure, a perfect fit to the metallicity distribution of the Geneva-Copenhagen Survey (Nordström et al. 2004, Holmberg et al. 2007) was possible if radial mixing was allowed for, and that no acceptable fit could be achieved without it. This new model of chemical evolution gives a completely different history of the disc compared to classical approaches. Since a large range of different galactocentric radii contribute their populations to local datasets, there is no need for the local star-forming interstellar medium to have followed the observed density ridges in the $[\alpha / \mathrm{Fe}],[\mathrm{Fe} / \mathrm{H}]$ plane. Among the most important differences between classical modelling without radial mixing and models including radial migration are the predicted correlations between chemistry and kinematics along the thin-disc ridge line. In larger datasets containing $[\alpha / \mathrm{Fe}]$ ratios, such as that of Borkova \& Marsakov (2005), thin-disc stars can be picked out from stars belonging to the thick disc and halo by selecting for low $[\alpha / \mathrm{Fe}]$ and dropping obvious halo stars. In this subsample one finds a highly significant increase of rotational velocity along the thin-disc ridge line moving from higher to lower metallicities, in nice concordance with the SB09 model. In the classical models the metal-poor stars should be the oldest thin-disc stars, so the increase in rotational velocity should be associated with an increase in velocity dispersion. The data do not show a significant increase towards lower metallicities, so the classical view is in conflict with the data.

\section{References}

Borkova, T. V. \& Marsakov, V. A. 2005 ARep, 49, 405

Holmberg, J., Nordström, B., \& Andersen, J. 2007 A $\& A$ A, 475, 519

Nordström, B., Mayor, M., Andersen, J., Holmberg, J., Pont, F., Jörgensen, B.R., Olsen, E. H., Udry, S., \& Mowlawi, N. 2004 A\&A, 418, 989

Schönrich, R. \& Binney, J 2009, MNRAS, 396, 203

Sellwood, J. A. \& Binney, J. 2002, MNRAS, 336, 785 